\title{
Cognitive Offloading: Can ubimus technologies affect our musicality?
}

\author{
Leandro Costalonga, Marcelo S. Pimenta \\ NESCoM - Universidade Federal do Espírito Santo \\ BR 101 Km 60 - 29932-540 São Mateus, ES \\ Instituto de Informática, Universidade Federal do Rio Grande do Sul \\ Caixa Postal 15064 - 90501-970 Porto Alegre, RS \\ leandro.costalonga@ufes,br, mpimenta@inf.ufrgs.br
}

\begin{abstract}
An interaction design that lean towards musical traits based on and constrained by our cognitive and biological system could, not only provide a better user experience, but also minimize collateral effects of excessive use of such technology to make music. This paper presents and discuss innate abilities involved in musical activities that - in the authors' viewpoint - could be considered in design guidelines to computer music technologies, especially those related to ubimus.
\end{abstract}

\section{Introduction}

If we consider music as a product of musicality, a social and cultural construct of humankind based on the presence of several cross-cultural similarities, it is very important to investigate how such musicality is affected by the technology we are using and building to provide support to our musical activities. We think everyone is a skilled and sophisticated musical listener, even those who consider themselves to be "unmusical" or do not themselves produce music have an implicit knowledge of the musical forms and styles of their culture (even if they cannot be expressed explicitly). For instance, all individuals have an implicit understanding of the melodic, rhythmic, and harmonic regularities of their cultures' music, in the same way they unconsciously know the structure and rules of our native language [1].

The growing man-machine integration results in a broader understanding of human experience and cognition. The same way that McLuhan has discussed how communication technology (alphabetic writing, the printing press, and the electronic media) affects cognitive organization, we think the use of ubimus technology could also shape the way we think about music or even have any (positive or detrimental) effect on our musicality.

The ability to rely on the external mind might have detrimental consequences to cognition [2] because humans are "cognitive misers", meaning that people tend to eschew costly analytic thought in favour of comparatively effortless intuitive processing [3]. The miserly nature of human cognition lends itself to an overreliance on simple heuristics and mental shortcuts [4, 5]. The evidence suggests that high smartphones' users are genuinely lower in cognitive ability and have a more intuitive cognitive style. Based on that premise, we are investigating if the use of ubimus technology could also shape the way we think music or has any detrimental effect on our musicality. This paper presents and discuss aspects that could improve the design guidelines to computer music technologies, specially related to ubimus.

For instance, Sparrow \& Wegner [6] pointed out that when people expect to have future access to information, they have lower rates of recall of the information itself and enhanced recall for where to access it instead. In a musical context, it is known that shortterm memory capacity is crucial to the segmentation strategy used by good sightreaders whenever reading a musical score [7, 8]. Sight reading is especially important in the first stage of the musical performance plan, that is acquiring knowledge of the music and developing preliminary ideas about how it should be performed. According to Gabrielsson [9], it is also in this first stage that the structural 
analysis reveals the real meaning of the musical information. Such a cognitively demanding task requires a substantial amount of analytical reasoning that, in turn, can be ultimately trusted to our smartphones as demonstrated by Barr et al. [3]. The second stage - the musical performance plan - involves hard work on technical problems in order to establish the spatiomotor pattern required to perform the music. Finally, the third and final stage is a fusion of the two previous stages with trial rehearsals that produces a final version of the performance [9]. The last two stages above mentioned demands executive functioning and anxiety control and yet, once again, the dependence on the smart devices plays a significant disrupting role at performing this task $[10,11]$.

Despite the evidences, it would be premature to state that the very best technology that has been created in order to provide support to musical activities purpose is, in fact, atrophying our musicality. Nevertheless, it is possible to approach this issue from a different angle, that is, researching about the cognitive and biological traits involved in musical thinking and applying it in the design of new ubimus tech. Doing so, we would lean towards innate and primitive structures related to music making, which is unlikely to change due to behavioral overuse of these technologies.

If musicality can be defined as a natural, spontaneously developing set of traits based on and constrained by our cognitive and biological system, music in all its variety can be defined as a social and cultural construct based on that very musicality [12], as will be discussed in the next section.

\section{Musicality: Cognitive and Biological Musical Traits}

We all can perceive and enjoy music. Over the years, it has become clear that all humans share a predisposition for music, just like we have for language. To recognize a melody and perceive the beat of music is an example of a trait based on and constrained by our cognitive abilities and their underlying biology (trivial skill for most humans). Even infants are sensitive to such features, which are common across cultures $[13,14]$. Other common human traits in musicality reported by Honing [1] are : a) relative pitch (e.g., contour and interval analysis; b) regularity and beat perception; c) tonal encoding of pitch; and d) metrical encoding of rhythm.

Until relatively recently, most scholars were wary of the notion that music cognition could have a biological basis. Music was viewed as a cultural product with no evolutionary history and no biological constraints on its manifestation. This explanation is supported by the belief that music has not been around long enough to have shaped perceptual mechanisms over thousands of generations. Moreover, in contrast to speech, this musical knowledge is acquired relatively slowly and not equally by all individuals of a given nature [15]. Such notions, however, do not explain the presence of music in all cultures and time periods, let alone other species. More recently, studies have indicated that our capacity for music has an intimate relationship with our cognition and underlying biology, which is particularly clear when the focus is on perception rather than production $[1,16,17]$.

Comparative research shows that although music itself may be specifically human, some of the fundamental mechanisms that underlie human musicality are shared with other species. For Darwin, music had no survival benefits but offered a means of impressing potential partners, thereby contributing to reproductive success. If so, possibility these cognitive traits are the target of natural selection (bear in mind that cognitive traits are polygenic). Darwin even argued that musical vocalizations preceded language [18].

Impressing potential partners may be a feasible purpose for music, however there are divergent studies on that matter. Other reported purposes for music are: a) promotion and maintenance of group cohesion, working as a glue that enhances cooperation and strengthens feelings of unity [19]; b) ease the burdens of caregiving and promote infant well-being and survival [20]. This view even see such vocalizations as having paved the way not only for language but also for music [21] and c) music is a technology or transformative invention that makes use of existing skills and has consequences for culture and biology [22], 
While there might be quite some evidence that components of musicality overlap with non-musical cognitive features, this is in itself no evidence against musicality as an evolved biological trait or set of traits. It still has to be demonstrated that the constituent components of musicality, when identified, are indeed domain specific. As in language, musicality could have evolved from existing elements through evolutionary processes, such as natural or sexual selection. Alternatively, based on the converging evidence for music-specific responses along specific neural pathways, it could be that brain networks that support musicality are partly recycled for language, thus predicting more overlap than segregation of cognitive functions.

All in all, consensus is growing that musicality has deep biological foundations, based on accumulating evidence for the involvement of genetic variation [23, 24]. Recent advances in molecular technologies provide an effective way of exploring these biological foundations, such as the association studies of genome aiming to capture the polymorphic content of a large phenotyped population sample.

\section{Guidelines: Interaction and UbiMus}

So far, based on the referred literature, it has been stablished that: a) there might be cognitive and biological traits related to musical activities; b) some human cognitive skills could be affected by ubiquitous technology, especially connected mobile devices. The question that is now posed is: how to design better ubiquitous technology for musical activities (UbiMus) that makes the most of our innate predisposition to music (musicality) in order to minimize the detrimental cognitive effects of extensive use of such devices?

Historically, digital things made by interaction designers were largely tools intended to be used instrumentally, for solving problems and carrying out tasks, and mostly to be used individually. In this scenario, concepts such as user goals, task flows, usability and utility were (and still are) very valuable. However, it turns out that digital technology in today's society is mostly used for communication (many-to-many), entertainment, and for pleasure. This is where user experience design thrives.

As the name suggests, user experience design is about designing the ideal experience of using a service or product. It is about the way people feel about a product and their pleasure and satisfaction when using it, looking at it, holding it, etc. Every product that is used by someone has a user experience. There are numerous theories, methodologies, and frameworks that help designers to design products focused on the user experience. It is not in the scope of this work to discuss it; however, they all suggest paying close attention to the user's needs and expected behavior (known as User-Centered Design). The user must be in the center of the designing process, they must not only be listened but also be involved. Overall, it is essential to take into account what people are good and bad at, both in a motor and cognitive level. For that reason, Human Computer Interaction (HCI) has been always interconnected with the fields or ergonomics and cognitive sciences [25]. Next, some important aspects of human cognition related to music are presented aiming to guide the development of new computer music technology.

\subsection{Establish a reference to be imitated}

(True) imitation is innate. It is welldeveloped in humans being observed in newborns babies both for fostering learning and for yielding pleasure. There is a distinction between imitation that copies the task structure and hierarchical organization, and imitation that copies movements. True imitation focuses on the goal, in other words, the execution of the action as a function of the goal[26]. In a musical context, can be approached from different viewpoints, such as: imitation skills, musical figures, imitation of symbols, imitation of moving sonic forms (corporeal imitation), and imitation of group behaviour (alleloimitation).

Playing a musical instrument starts with the imitation of low-level skills and low-level challenges. However, as skills improve, the challenges can rise to a higher level. When skills and challenges are in equilibrium, this gives rise to an optimal experience or pleasure. 
Learning to play a musical instrument is, therefore, a typical example of true imitation. It draws on the ability of the student to focus on what is essential in the teacher's example. Even if the instrument is not the same it is still possible to imitate particular behaviours and playing styles because the student has more of a focus on the goals and less of a focus on the precise movements. However, the student's ability to see the movements and gestures of the teacher may be an important component in learning to play a musical instrument. The visual observation of expressive movements may facilitate the mirroring of the teacher's intentions to the student's intentions [27].

The role of mirroring in music education has been confirmed by a brain imaging study [28] . Playing of a musical instrument was used to show that the decomposition into elementary motor components was encoded by the mirror neurons. When the action to be imitated corresponded to an elementary action already present in the mirror neuron system, this act was forwarded to other structures and replicated. In that case, no learning was needed [27].

The conclusion here is that, in order to capitalize on the human innate capability to imitate, the designers of a computational performance tool must take into account ways of facilitating this process of true imitation either by providing key examples as well as awareness of the performer on actions compared to others. Guidance and a reference is needed.

\subsection{Building blocks}

Barr et al. [3] studies suggest that people who think more intuitively and less analytically when given reasoning problems were more likely to rely on their connected devices, suggesting that people may be prone to look up information that they actually know or could easily learn, but are unwilling to invest the cognitive cost associated with encoding and retrieval. In that sense, a possible approach is to offer building blocks that simplifies a set of complex tasks that can looked closer whenever the user feels prepared to do so. Those abstractions can represent performer's actions, musical structures (i.e. arpeggios), emotional intention, improvisation strategy (with the use of AI), riffs, samples, rhythmic patterns, etc. Not only these blocks should be easily available, searchable, but it should also be suggested based on context and user profiling.

Note, however, that music is still believed to be mostly a matter of the "intuitive" right brain - the avatar of emotion and creativity [15]. If that so, chances are the user will never look into the building blocks since one might choose not to engage in costly elaborative encoding, as they know that knowledge can be procured externally. Therefore, besides being a good approach to manage frustration and anxiety, building blocks strategy might not be ideal, for example, for musical learning software.

\subsection{Movements and Gestures}

As previously mentioned, people engage with music in a way similar to the way they engage with other people[27] : the process of an appreciation of music - although including also cerebral appreciation and interpretation -is strongly based on mirroring body movement.

In order to sound natural in performance, expressive timing must conform to the principle of human movement [29]. People's tendency to move in synchrony with auditory rhythms is known as ideomotor principle: perception of movement will always induce a tendency to perform the same or similar movements [30]. The effect is clearly observable in the tendency to tap along with the beat of the music [27]. The beat is the most natural feature for synchronized movement because it appeals to fundamental biomechanical resonances [31]. In this regard, Knuf et. al. [30] ran a comprehensive study on ideomotor actions and verified that movements did not always occur without awareness, but they did occur without awareness of voluntary control. They have also found clear evidence that people do tend to perform the movements they would like to see (intentional induction) whereas results are less clear with respect to perceptual induction (movements that people actually see). Perceptual induction could only be verified thru noninstrumental effectors: in their experiment, the effect appeared for both head and foot. For hand movements (the instrument effectors), intentional induction is 
much more pronounced than perceptual.

Corporeal articulation is also related to musical expressiveness and can be seen as indicators of intentionality (studied as seen above in terms of this mirroring process)[27]. In general terms, movement in response to music is often seen as a gestural expression of a particular emotion (sadness, happiness, love, anger) that is assumed to be imitated by the music [32]. Therefore, a fundamental question is: if articulations are a kind of expression, how do they relate to expressiveness in music? [27] Bear in mind that the expression of emotion is only one aspect of corporeal involvement with music since corporeal articulations can also be used to annotate structural features, such as melody, tonality, and percussion events. In summary, our gestures and movements give away our intentions and must be considered when musical activities take place.

\subsection{Tempo, timing, regularity}

Todd [33] defends the principle that performance, perception of tempo and musical dynamics are based on an internal sense of motion. This principle reflects upon the notion that music performance and perception have their origins in the kinematic and dynamic characteristics of typical motor actions. For example, regularities observed in a sequence of foot movements during walking or running are similar to regularities observed in sequences of beats or note values when a musical performance changes tempo.

A shared assumption from these lines of works is that we experience and make sense of musical phenomena by metaphorically mapping the concepts derived from our bodily experience of the physical world into music. Accordingly, listeners hear the unfolding musical events as shaped by the action of certain musical forces that behave similarly to the forces behind our movements in the physical world such as gravity and inertia[34]. Baily [35] even argues that the performer's internal representation of music is in terms of movement, rather than sound.

Honing [36] defends that regularity and beat perception is one of the human's innate musical traits. Cate et al. [37] goes beyond studding in different species both the ability to recognize the regularity in the auditory stimulus and the ability to adjust the own motor output to the perceived pattern. Although rare, this ability appears to some animals as well.

Human rhythmic abilities obviously did not arise to allow people to synchronize to metronomes but rather to the actions of other humans in groups, known as social synchronization. Thus, by the ecological principle, the concept of mutual entrainment among two or more individuals should be the ability of central interest rather than BPS to a mechanical timekeeper [38]. In the wild (i.e., outside the lab), the mutual entrainment of two or more individuals by such a mechanism obviously cannot occur unless they themselves are capable of producing the evenly paced entraining stimulus of some kind (such as clapping, stomping, or drumming) within the tempo range of its predictive timing mechanism [39].

The most sophisticated form of synchronization involves beat-based predictive timing, where an internal beat is tuned to the frequency and phase of an isochronous timegiver, allowing perfect 0 -degree phase alignment. This stimulus makes the very next beat in the sequence predictable, allowing the timing mechanism to align - or latch-its produced behavioral to the stimulus with zero, or even small negative (anticipatory), phase lag, typical of human sensorimotor synchrony [40]. Because of reaction time limitations, it cannot therefore be based on responding to that stimulus event. Instead, it requires a predictive (anticipatory) and cyclical motor timing mechanism that takes an evenly paced stimulus sequence as input. Naturally, reaction times to predictable stimuli are shorter than those to unpredictable ones, hence preparatory cues such as "ready, steady" or a drummer's count down allow quicker responses.

\subsection{Tonal hearing}

Humans readily recognize tone sequences that are shifted up or down in log frequency because the pattern of relative pitches is maintained (referred to as interval perception or relative pitch). Humans also have sensitivity to spectral contour of musical signals (like birds) but relative pitch is the preferred mode of 
listening for humans.

The tonal encoding of pitch also seems to be another innate human ability regarded to music. There is substantial empirical evidence that listeners use this tonal knowledge in music perception automatically. Central to pitch organization is the perception of pitch along musical scales. A musical scale refers to the use of a small subset of pitches in a given musical piece. Scale tones are not equivalent and are organized around a central tone, called the tonic. Usually, a tonal musical piece starts and ends on the tonic. Tonal organization of pitch applies to most types of music, but it does not occur in processing other sound patterns, such as speech. Although the commonly used scales differ somewhat from culture to culture, most musical scales use pitches of unequal spacing that are organized around 5-7 focal pitches and afford the building of pitch hierarchies. The tonal encoding module seems to exploit musical predispositions, as infants show enhanced processing for scales with unequal pitch steps [37].

Innate musical predispositions implies specialized brain structures to deal with music: tonal encoding can be selectively impaired by brain damage; for example, after damage some patients are no longer able to judge melodic closure properly. Recent functional neuroimaging study has identified the rostromedial prefrontal cortex as a likely brain substrate for the 'tonal encoding' module [38]. Musical memory is also organized, at least partially, independent of the hippocampus - the brain structure that is central to memory formation. It is possible that the enormous significance of music throughout all times and in all cultures contributed to the development of an independent memory for music [39].

After all these findings, we need to rethink the specificity of music related brain cells may be not fully exploited by the traditional (ubimus) design decisions.

\section{Final Discussion}

Music, as we know it, has been done in the same way for centuries. It is true that musical instruments have evolved to provide a better (not ideal) fit to our body, sound quality has been improved, new musical styles appeared while others died, and the musical industry reinvented itself several times over. Nevertheless, the cognitive and biological traits that support all this activity, from producing to enjoying music, has been largely the same. Evolution on this matter comes slow. Fact is, humans excel at doing music this way.

With the popularization of the computer technology, a lot of progress had to be made in field of HCI to allow the computers to be usable by nonspecialized regular people. Currently, computers (in all its modern shapes and forms) are involved in virtually all activities known by human beings, music included. A relevant change of paradigm in HCI was Weiser's [41] vision of ubiquitous technology (UbiComp). [25].The readings of UbiComp concepts and technology by fields such as Music and Arts led to the birth of subfield so called Ubiquitous Music (UbiMus); This field is concerned with ubiquitous systems of human agents and material resources that afford musical activities through creativity support tools. In practical terms, ubimus is music (or musical activities) supported by ubiquitous computing concepts and technology. It relates to concepts such as: portability, mobility, connectivity, availability (including for non-musicians).

More recently, another emerging research field positioned itself at the intersection of Internet of Things, new interfaces for musical expression, ubiquitous music, human-computer interaction, artificial intelligence, and participatory art - it is known as Internet of Musical Thing (IoMusT). From a computer science perspective, IoMusT refers to the networks of computing devices (smart devices) embedded in physical objects (musical things) dedicated to the production and/or reception of musical content. Possible scenarios for IoMusT are: augmented and immersive concert experiences; audience participation; remote rehearsals; music e-learning; and smart studio production [35].

These are very exciting research areas, but the big question is: are we cognitively equipped to make the most of it? Could we thrive in this new way of making music or it will become "yet-another" short-lived cool interface for music making? Is this new technology really paying attention to the way 
we do things, taking into consideration what we are good and bad at? Clearly, there is an intrinsic exploratory value in these initiatives, and it will certainly lead to unpredictable artistic outcomes, but this is insufficient to answer the questions above. Moreover, is there a price to pay when we rely so heavily in such tech to perform an activity such as music making?

One of the most pertinent questions for the 21 st century is how these increasingly intelligent and invasive technologies will affect our minds. The term "extended mind", has been used in order to refer to the notion that our cognition goes beyond our brains and suggests that individuals may allow their smart devices to do their thinking for or with them [3]. The ability to rely on the external mind might have detrimental consequences to cognition [2].

This paper discussed and presented some innate abilities involved in musical activities that - in the authors' viewpoint - could be considered in the current designs of digital musical instruments and ubimus technology as a whole. Even we need more experimental work, recent findings of biomusicology and neuroscience indicate musical activities may have idiossincrasies that are not covered by the traditional approaches of interaction design and user experience design.

\section{References}

1. Honing, H.: The origins of musicality. The MIT Press, Cambridge, Massachusetts, USA (2018)

2. Carr, N.: The Shallows: what the internet is doing to our brains. W.W. Norton \& Company, New York, New York, USA (2010)

3. Barr, N., Pennycook, G., Stolz, J.A., Fugelsang, J.A.: The brain in your pocket: Evidence that Smartphones are used to supplant thinking. Comput. Human Behav. 48, 473-480 (2015)

4. Kahneman, D.: Thinking, fast and slow. (2011)

5. Stanovich, K.E.: The robot's rebellion : finding meaning in the age of Darwin. University of Chicago Press (2004)

6. Sparrow, B., Liu, J., Wegner, D.M.: Google effects on memory: cognitive consequences of having information at our fingertips. Science. 333, 776-8 (2011)
7. Bean, K.L.: Reading music instead of spelling it. J. Musicol. 1-5 (1939)

8. Gabrielsson, A.: Music performance research at the millennium. Psychol. Music. 31, 221 (2003)

9. Gabrielsson, A.: The performance of music. Deutsch, Diana (1999). The psycho, 501-602 (1999)

10. Clayton, R.B., Leshner, G., Almond, A.: The Extended iSelf: The Impact of iPhone Separation on Cognition, Emotion, and Physiology. J. Comput. Commun. 20, 119-135 (2015)

11. Hartanto, A., Yang, H.: Is the smartphone a smart choice? The effect of smartphone separation on executive functions. Comput. Human Behav. 64, 329-336 (2016)

12. Honing, H., Ploeger, A.: Cognition and the Evolution of Music: Pitfalls and Prospects. Top. Cogn. Sci. 4, 513-524 (2012)

13. Savage, P.E., Brown, S., Sakai, E., Currie, T.E.: Statistical universals reveal the structures and functions of human music. Proc. Natl. Acad. Sci. U. S. A. $112,8987-92$ (2015)

14. Trehub, S.E., Becker, J., Morley, I.: Crosscultural perspectives on music and musicality. Philos. Trans. R. Soc. Lond. B. Biol. Sci. 370, 20140096 (2015)

15. Repp, B.H.: Some cognitive and perceptual aspects of speech and music. In: Music, Language, Speech and Brain. pp. 257-268. Macmillan Education UK, London (1991)

16. Fitch, W.T.: The biology and evolution of music: A comparative perspective. Cognition. 100, 173-215 (2006)

17. Winkler, I., Háden, G.P., Ladinig, O., Sziller, I., Honing, H.: Newborn infants detect the beat in music. Proc. Natl. Acad. Sci. U. S. A. 106, 2468-71 (2009)

18. Fitch, W.T.: Rhythmic cognition in humans and animals: distinguishing meter and pulse perception. Front. Syst. Neurosci. 7, 68 (2013)

19. Merker, B.H., Madison, G.S., Eckerdal, P.: On the role and origin of isochrony in human rhythmic entrainment. Cortex. 45, 4-17 (2009)

20. Dissanayake, E.: If music is the food of love, what about survival and reproductive success? Music. Sci. 12, 169-195 (2008) 
21. Brown, S.: Evolutionary Models of Music: From Sexual Selection to Group Selection. In: Thompson, F.T.S. (ed.) Perspectives in Ethology. pp. 231-281. Springer, Boston, MA, Boston, MA. (2011)

22. Patel, A.D.: Music, language, and the brain. Oxford University Press, New York, NY (2008)

23. Oikkonen, J., Onkamo, P., Järvelä, I., Kanduri, C.: Convergent evidence for the molecular basis of musical traits. Sci. Rep. 6, 39707 (2016)

24. Särkämö, T., Altenmüller, E., RodríguezFornells, A., Peretz, I.: Editorial: Music, Brain, and Rehabilitation: Emerging Therapeutic Applications and Potential Neural Mechanisms. Front. Hum. Neurosci. 10, 103 (2016)

25. Preece, J., Rogers, Y., Sharp, H.: Interaction design : beyond human-computer interaction. Wiley, Chichester, UK. (2015)

26. Byrne, R.W., Russon, A.E.: Learning by imitation: a hierarchical approach. Behav. Brain Sci. 21, 667-84; discussion 684-721 (1998)

27. Leman, M.: Embodied music cognition and mediation technology. MIT Press, Cambridge, Massachusetts (2008)

28. Buccino, G., Binkofski, F., Riggio, L.: The mirror neuron system and action recognition. Brain Lang. 89, 370-376 (2004)

29. Honing, H.: The final ritard: On music, motion, and kinematic models. Comput. Music J. 27, 66-72 (2003)

30. Knuf, L., Aschersleben, G., Prinz, W.: An analysis of ideomotor action. J. Exp. Psychol. Gen. 130, 779-798 (2001)

31. Hallam, S., Cross, I., Thaut, M.: The Oxford handbook of music psychology. Oxford University Press, Oxford, UK (2005)

32. Friberg, A., Sundberg, J.: Does music performance allude to locomotion? A model of final ritardandi derived from measurements of stopping runners. J. Acoust. Soc. Am. 105, 1469-1484 (1999)

33. Todd, N.P.M.: The dynamics of dynamics - a model of musical expression. J. Acoust. Soc. Am. 91, 3540-3550 (1992)

34. Dogantan-Dack, M.: The body behind music: Precedents and prospects. Psychol. Music. 34, 449464 (2006)
35. Baily, J.: Music structure and human movement. Music. Struct. Cogn. 237-58 (1985)

36. Honing, H.: Without it no music: beat induction as a fundamental musical trait. Ann. N. Y. Acad. Sci. 1252, 85-91 (2012)

37. ten Cate, C., Spierings, M., Hubert, J., Honing, H.: Can Birds Perceive Rhythmic Patterns? A Review and Experiments on a Songbird and a Parrot Species. Front. Psychol. 7, 730 (2016)

38. Fitch, W.T.: Four principles of bio-musicology. Philos. Trans. R. Soc. B Biol. Sci. 370, $20140091-$ 20140091 (2015)

39. Merker, B., Morley, I., Zuidema, W.: Five fundamental constraints on theories of the origins of music. Philos. Trans. R. Soc. B Biol. Sci. 370, 20140095-20140095 (2015)

40. Fraisse, P.: Rhythm and tempo. Psychol. Music. 2, 149-180 (1982)

41. Weiser, M.: The Computer for the $21 \mathrm{st}$ Century. Sci. Am. 265, 94-105 (1991) 